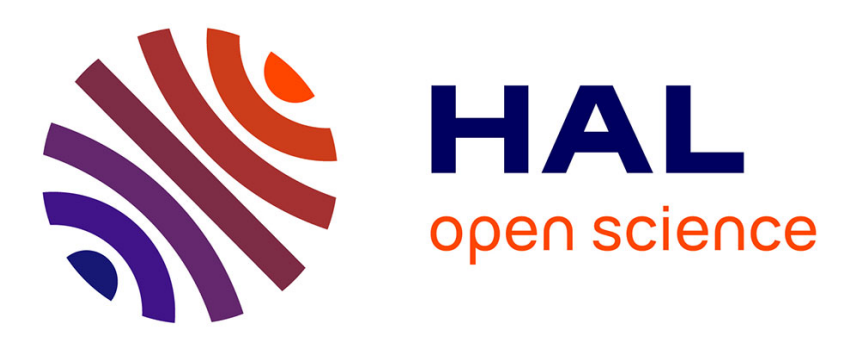

\title{
Rayonnement électromagnétique des décharges orageuses. Analyse submicroseconde
}

\author{
Ch. Leteinturier, J. Hamelin
}

\section{To cite this version:}

Ch. Leteinturier, J. Hamelin. Rayonnement électromagnétique des décharges orageuses. Analyse submicroseconde. Revue de Physique Appliquée, 1990, 25 (2), pp.139-146. 10.1051/rphysap:01990002502013900 . jpa-00246172

\section{HAL Id: jpa-00246172 https://hal.science/jpa-00246172}

Submitted on 1 Jan 1990

HAL is a multi-disciplinary open access archive for the deposit and dissemination of scientific research documents, whether they are published or not. The documents may come from teaching and research institutions in France or abroad, or from public or private research centers.
L'archive ouverte pluridisciplinaire HAL, est destinée au dépôt et à la diffusion de documents scientifiques de niveau recherche, publiés ou non, émanant des établissements d'enseignement et de recherche français ou étrangers, des laboratoires publics ou privés. 


\title{
Rayonnement électromagnétique des décharges orageuses. Analyse submicroseconde
}

\author{
Ch. Leteinturier $\left({ }^{(}\right)$et J. Hamelin ( $\left.{ }^{2}\right)$ \\ (1) Centre National d'Etudes des Télécommunications, France Télécom, Lannion, France \\ (2) Agence Spatiale Européenne, E.S.A./ESTEC, Noordwijk, Pays-Bas
}

(Reçu le 27 février 1989, révisé le 28 avril 1989, accepté le 2 mai 1989)

\begin{abstract}
Résumé. - Les champs électromagnétiques rayonnés par les décharges orageuses ont été caractérisés en formes et amplitudes, et plus particulièrement les processus rayonnant jusqu'à quelques dizaines de $\mathrm{MHz}$, à savoir les arcs-en-retour, les décharges intranuages et certains précurseurs. Les dernières mesures ont montré que les champs accompagnant les décharges, qu'elles soient naturelles ou déclenchées, présentent toutes de larges variations submicrosecondes quand l'atténuation de l'onde de sol est minimale. Dans le cas des éclairs déclenchés, nous avons pu corréler les transitions submicrosecondes du champ électromagnétique rayonné à proximité de la décharge à celles du courant lui-même. De cette corrélation, nous pouvons en déduire que la vitesse de propagation de l'onde de courant le long du canal est beaucoup plus grande à sa base (de l'ordre de 2 à $2,5 \times 10^{8} \mathrm{~m} / \mathrm{s}$ ) qu'à plusieurs centaines de mètres ou un ou deux kilomètres au-dessus du sol (de l'ordre de $\left.10^{8} \mathrm{~m} / \mathrm{s}\right)$.
\end{abstract}

\begin{abstract}
The electromagnetic fields radiated by the lightning discharges have been characterized in waveforms and magnitudes, more particularly the return-strokes, the intracloud discharges and some leaders which radiate up to a few tens of MHz. The last measurements showed that the E.M. fields produced by lightning discharges, natural or triggered, all present large submicrosecond transitions when the ground wave attenuation is minimum. When the lightning discharges were triggered, it was possible to correlate the submicrosecond variations of the electromagnetic field radiated at a close range from the stroke impact point to the current itself. From this correlation, it was possible to deduce that the upward propagation velocity of the current wave is much larger near the ground (of the order of 2 to $2.5 \times 10^{8} \mathrm{~m} / \mathrm{s}$ ) than a few hundreds of meters or one or two kilometers above ground (of the order of $10^{8} \mathrm{~m} / \mathrm{s}$ ).
\end{abstract}

L'examen des champs électromagnétiques accompagnant une décharge orageuse est la seule approche expérimentale possible des phénomènes électriques liés à cette décharge, à l'exception des éclairs déclenchés pour lesquels, en maîtrisant à la fois l'instant et le lieu de la décharge, il est possible de mesurer le courant de l'éclair.

Dans cette démarche, les premiers points étudiés furent les phénomènes de grande amplitude liés aux arcs-en-retour des décharges nuage-sol (rappelons que le courant de retour parcourant le canal arc créé par les traceurs est de quelques dizaines de kiloampères). Le canal de l'éclair, assimilable à une antenne verticale de quelques kilomètres, est parcouru par cette impulsion de courant. La résultante est alors une impulsion électromagnétique très intense ; à proximité de l'éclair: de l'ordre de $4000 \mathrm{~V} / \mathrm{m}$ à
$200 \mathrm{~m}$ et $100 \mathrm{~V} / \mathrm{m}$ à $3 \mathrm{~km}$; le champ rayonné à $50 \mathrm{~km}$ est de l'ordre de $4 \mathrm{~V} / \mathrm{m}$; ces valeurs correspondent à un courant crête de $10 \mathrm{kA}$.

Ces décharges, parcourant un canal préalablement ionisé, qu'elles soient dues à un processus entre nuage et sol ou intranuage, ont un rayonnement électromagnétique important dans les premières dizaines de $\mathrm{MHz}$. De même, certains phénomènes précurseurs (par exemple, les précurseurs par pas) rayonnent dans cette bande de fréquence. Les phénomènes liés aux rayonnements VHF-UHF correspondent essentiellement aux processus initiateurs des décharges, tant à l'intérieur du nuage que lors de l'avancée des précurseurs dans le canal arc.

Nous nous intéresserons au rayonnement dans la partie basse du spectre, soit $200 \mathrm{~Hz}-100 \mathrm{MHz}$ (décharges nuage-sol et intranuage), et plus particu- 
lièrement à leur front de montée par l'analyse des signaux $\mathrm{d} E / \mathrm{d} t$. Enfin, pour le cas des décharges nuage-sol déclenchées, nous étudierons la relation entre les transitions rapides du courant de foudre et celles du champ électromagnétique associé.

\section{Caractéristiques du rayonnement électromagnétique (E.M.) impulsionnel des décharges orageuses.}

L'observation continue du rayonnement E.M. (dans la bande $150 \mathrm{~Hz}-20 \mathrm{MHz}$ ) pendant une période orageuse a permis de mettre en évidence que, pendant quelques centaines de millisecondes précédant une décharge nuage-sol (ou même en l'absence de décharge nuage-sol), nombre d'impulsions dans le nuage pouvaient apparaître, soit « isolées » avec un temps de séparation de quelques millisecondes ou dizaines de millisecondes, soit au contraire par paquets de quelques dizaines, séparées les unes des autres de quelques microsecondes ou dizaines de microsecondes. Ces processus peuvent également se produire après les décharges nuage-sol. Des mesures optiques visant à $5^{\circ}$ ou $10^{\circ}$ au-dessus de l'horizon ont permis de discerner les éclairs nuage-sol.

L'enregistrement simultané des signaux HF utilisant des capteurs magnétiques large bande, et des signaux VHF-UHF, a permis d'établir une phénoménologie macroscopique des décharges. La figure 1 représente quelques signaux de champ magnétique rayonnés par les différentes phases d'un éclair, à l'aide de capteurs magnétiques $150 \mathrm{~Hz}-20 \mathrm{MHz}$, enregistrés sur bande magnétique (B.P. $3 \mathrm{MHz}$ ).

La phase initiale qui précède le premier arc-enretour a duré plus de 500 millisecondes. Au cours de cette phase précédant les arcs-en-retour, des impulsions magnétiques brèves, unipolaires et bipolaires, caractéristiques des processus intranuage (Weidman, Krider, 1979) ont été observées par séquences. La figure 1a représente les impulsions E.M. avec les échelles de temps $50 \mu \mathrm{s}$ et $500 \mu \mathrm{s} / \mathrm{div}$, apparaissant

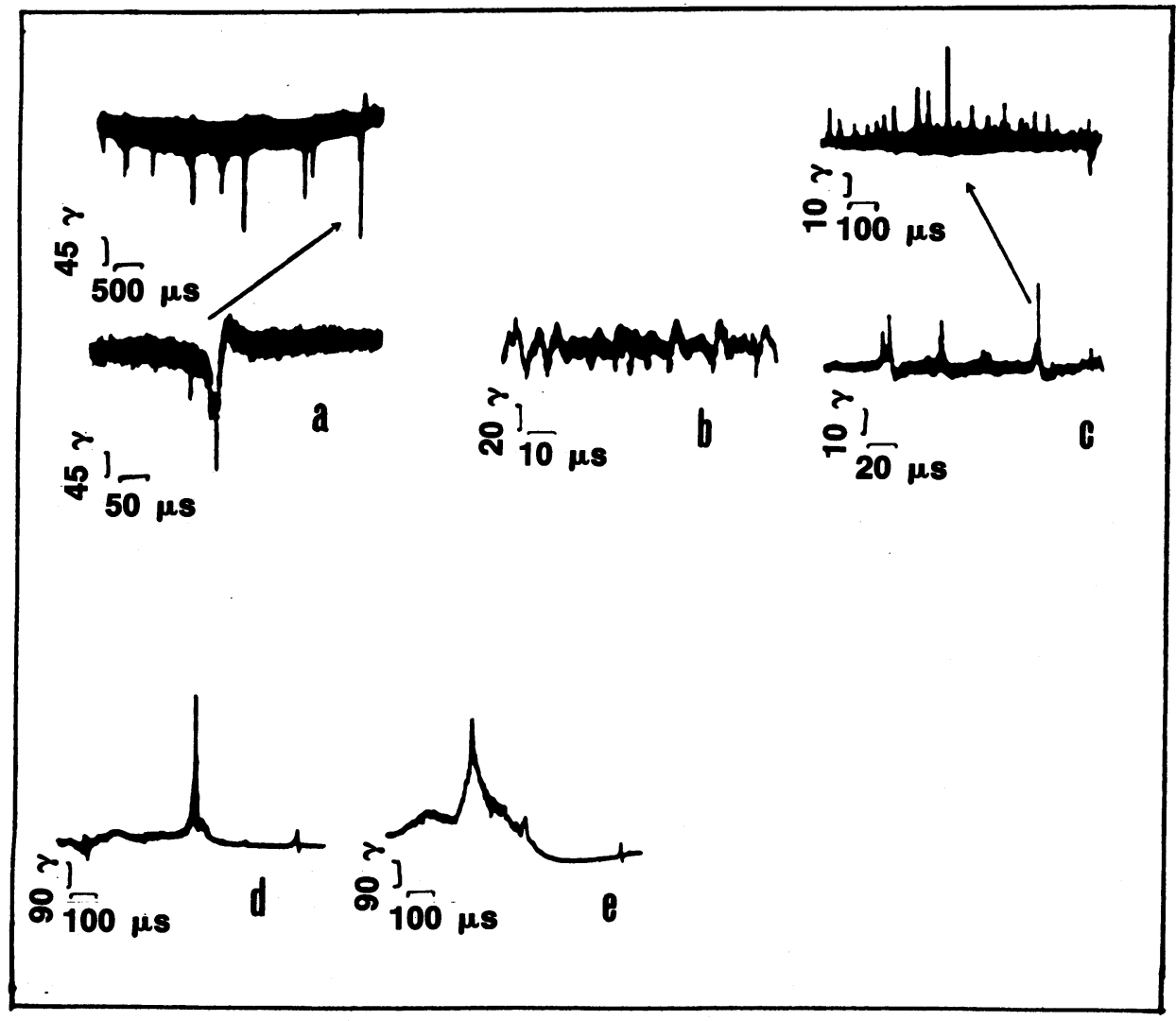

Fig. 1. - Signaux de champ magnétique enregistrés au cours d'un éclair naturel. (a) Impulsions intranuage, $500 \mathrm{~ms}$ avant le $1^{\text {er }}$ arc-en-retour, (b) impulsions intranuage bipolaires, $200 \mathrm{~ms}$ avant le $1^{\text {er }}$ arc-en-retour, (c) impulsions unipolaires liées au précurseur par pas précédant le $1^{\text {er }}$ arc-en-retour, (d) et (e) signatures du champ magnétique rayonné par le $1^{\text {cr }}$ et le $3^{\mathrm{e}}$ arcs-en-retour $-1 \gamma=10^{-9} \mathrm{~T}$. Les échelles de temps sont différentes selon les impulsions. (a) et (c) sont présentées avec 2 échelles de temps (Leteinturier et al., 1984).

[Magnetic field signals recorded during natural lightning. (a) Intracloud pulses, $500 \mathrm{~ms}$ before the first return-stroke, (b) bipolar intracloud pulses, $200 \mathrm{~ms}$ before the first return-stroke, (c) unipolar pulses produced by the stepped-leader before the first return-stroke, (d) and (e) magnetic field waveforms radiated by the 1 st and the $3 \mathrm{~d}$ return-strokes ; $1 \gamma=10^{-9} \mathrm{~T}$. The time scales are different from one pulse to an other. (a) and (c) are represented with two different time scales (Leteinturier et al., 1984).] 
aux premiers instants de la décharge, c'est-à-dire plus de $500 \mathrm{~ms}$ avant le premier arc-en-retour. D'autres petites impulsions sont apparues par séquences (Fig. 1b). Une dizaine de millisecondes avant la première décharge nuage-sol, des impulsions magnétiques unipolaires (Fig. 1c) séparées par quelques dizaines de microsecondes ont été enregistrées ; elles correspondent aux bonds du précurseur par pas ; en effet, le temps qui les sépare est de l'ordre du temps de pause entre deux bonds (Uman, 1969), et la durée totale similaire à celle de la progression du précurseur dans le canal. Ensuite, chaque arc-enretour a été accompagné d'impulsions magnétiques de grande amplitude (Figs. 1d et e).

Nous pouvons classer les impulsions de champ magnétique enregistrées avec quelques $\mathrm{MHz}$ de bande en trois grandes catégories, chacune d'elles étant représentée sur la figure 2. Les impulsions (1) et (2) sont caractéristiques des décharges intranuages, et l'impulsion (3) des décharges nuage-sol. L'impulsion (1) a une durée de l'ordre de 20 à $30 \mu \mathrm{s}$, elle est caractérisée par sa double polarité. L'impulsion (2), dite unipolaire, dure quelques microsecondes ; les impulsions de type unipolaire apparaissent par paquets, séparées de quelques microsecondes ; leur nombre varie de quelques unités à plusieurs dizaines. Nous avons fréquemment observé la présence d'impulsions unipolaires (2) précédant de 10 à $20 \mu$ s l'impulsion bipolaire (1). Ces impulsions (2) sont caractéristiques de processus à l'intérieur du nuage, mais également des précurseurs par pas qui précèdent le premier arc-en-retour.

L'impulsion (3) caractérise les décharges entre le nuage et le sol. La durée de cette impulsion est plus longue. Le pic initial est comparable à une impulsion

B(r)

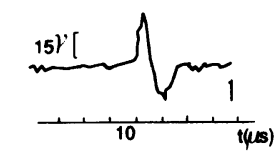

B(P)

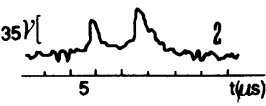

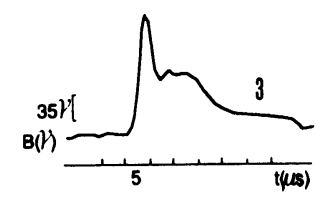

t(us) de type (2), mais la décroissance du signal est plus lente.

L'amplitude du champ E.M. rayonné par les processus intranuage est plus faible que celui des décharges nuage-sol mais cependant non négligeable. Remarquons sur la figure 1 que les amplitudes atteintes par les impulsions magnétiques rayonnées par la partie initiale de la décharge (a) sont pratiquement la moitié de celles liées aux arcs-en-retour ( $\mathrm{d}$ et e).

Remarquons également la fréquence d'apparition des impulsions E.M. liées aux processus intranuages. Celles-ci sont séparées de quelques dizaines de microsecondes, voire même de quelques microsecondes. Les impulsions liées aux décharges nuage-sol sont elles, séparées de plusieurs dizaines de millisecondes.

\section{Caractérisation submicroseconde du rayonnement électromagnétique.}

Au cours de plusieurs campagnes expérimentales, nous avons également caractérisé la dérivée temporelle des signaux de champ électrique rayonnés par les décharges naturelles et déclenchées. Dans le cas des décharges naturelles, nous avons pris en compte celles qui ont été localisées et dont la propagation électromagnétique se faisait au-dessus d'un sol de bonne conductivité, l'eau de mer.

Les signaux de champ électrique $E$ et de sa dérivée temporelle $\mathrm{d} E / \mathrm{d} t$ étaient mesurés avec des antennes plates capacitives, installées sur le toit d'une remorque métallique mise à la terre. Les capteurs et les systèmes électroniques furent définis de façon que le temps de réponse pour les mesures $\mathrm{d} E / \mathrm{d} t$ et $E$ soient minimum; ils ont été mesurés respectivement 10 et 125 ns. Les capteurs utilisés, et la détermination du facteur d'intensification du champ due à la remorque, sont décrits par Leteinturier et al. (1985).

Le synoptique de la chaîne d'acquisition utilisée en 1985 est donné sur la figure 3. Les acquisitions des signaux $E$ et $\mathrm{d} E / \mathrm{d} t$ étaient déclenchées, soit par le signal $\mathrm{d} E / \mathrm{d} t$ lui-même, soit par un signal de rayonnement $\mathrm{HF}$. Les signaux $\mathrm{d} E / \mathrm{d} t$ étaient échantillonnés à $100 \mathrm{MHz}$ et les signaux $E$ à $10 \mathrm{MHz}$. Une antenne capacitive supplémentaire permettait de connaître les variations plus lentes du champ électrique pendant toute la durée d'un éclair. Une synchronisation temporelle précise fut assurée entre les différentes mesures.

La mesure des signaux $\mathrm{d} E / \mathrm{d} t$ a montré que les décharges nuage-sol, les décharges intranuages et les précurseurs étaient accompagnés de larges transitions électromagnétiques submicrosecondes. Le tableau (Fig. 4) donne les valeurs normalisées à $100 \mathrm{~km}$ (décroissance en $1 / R$ du champ rayonné) pour les différents processus de décharges nuage-sol,
[Magnetic field pulses examples recorded during a natural lightning, including intracloud and cloud to ground discharges. The bipolar (1) and unipolar (2) pulses are produced by intracloud discharges. They are short and more frequent than pulses like (3) typical from cloud-toground flashes.]
Fig. 2. - Exemples d'impulsions magnétiques enregistrées au cours d'un éclair naturel comprenant des decharges type bipolaire (1) et unipolaire (2) sont des caractéristiques fréquentes que les impulsions (3) typiques des arcs-enretour (Leteinturier, Hamelin, 1984). 


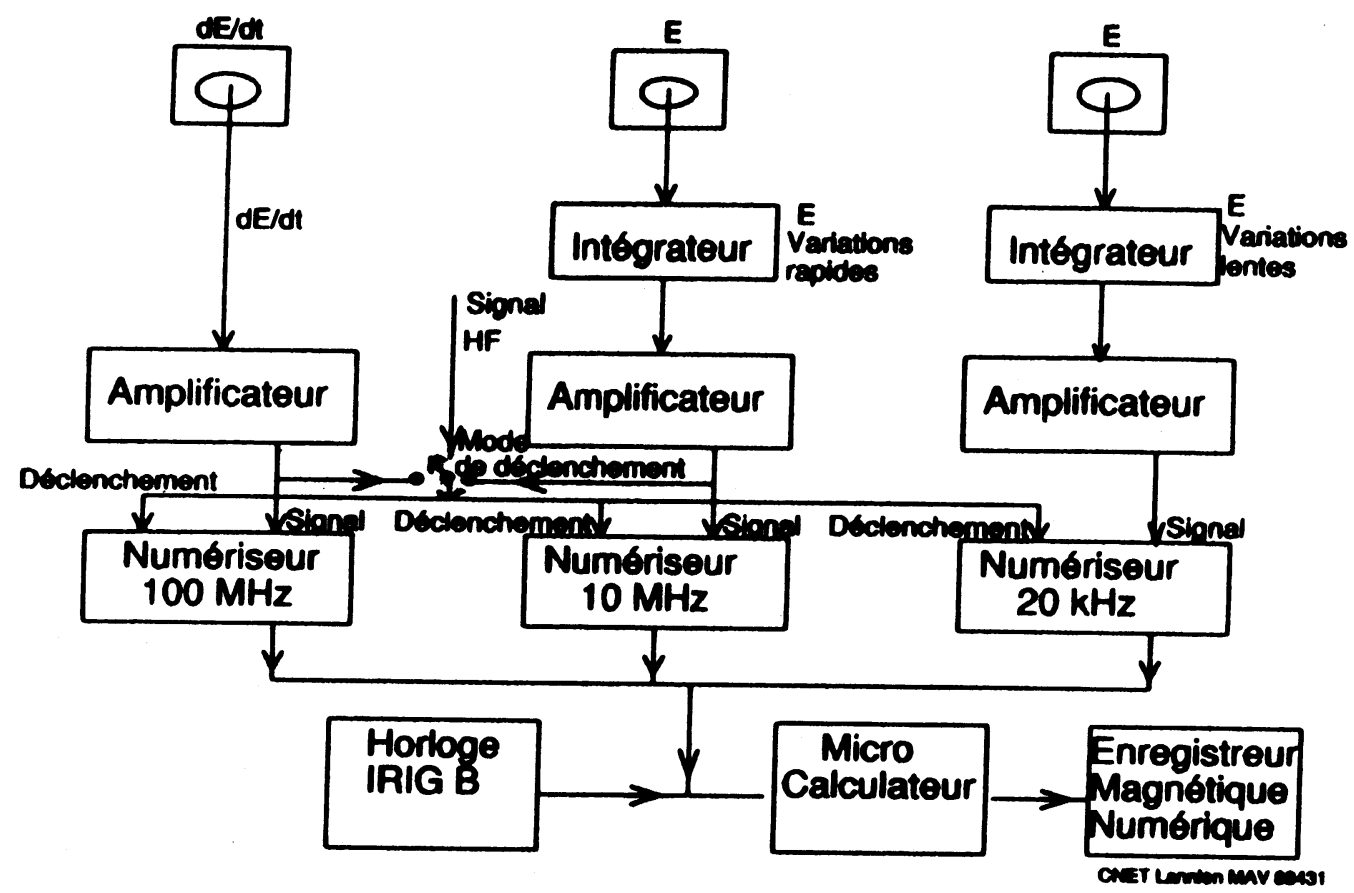

Fig. 3. - Synoptique du système expérimental utilisé en 1985 : capteurs et moyens d'acquisition.

[Block schematic diagram of the experimental means used in 1985 ; sensors and recording equipment.]

pour les décharges intranuages et pour les précurseurs par pas, les valeurs crête du champ électrique $E$, celles de la dérivée temporelle du champ électrique $\mathrm{d} E / \mathrm{d} t$ et la durée à mi-amplitude de ces signaux. On rappelle sur la figure 5 la différence des signaux de champ électrique associés aux premiers arcs-en-retour ou aux suivants.

La figure 6 représente l'enregistrement simultané de signaux $E$ et $\mathrm{d} E / \mathrm{d} t$ rayonnés par une décharge nuage-sol localisée sur l'océan à $36 \mathrm{~km}$ du site de mesures. La figure 7 représente l'enregistrement (a) du signal $\mathrm{d} E / \mathrm{d} t$ mesuré à $50 \mathrm{~m}$ d'un éclair déclenché et le signal $E$ (b) obtenu par calcul intégral de $\mathrm{d} E / \mathrm{d} t$. Un autre exemple de ce type est donné sur la figure 8, mais cette fois-ci, c'est l'enregistrement simultané des signaux $E$ (b) et $\mathrm{d} E / \mathrm{d} t$ (a) à $5 \mathrm{~km}$ de l'éclair déclenché.

Relation entre le courant de foudre et le rayonnement
électromagnétique.

La technique de déclenchement des éclairs nous a donné accès aux mesures du courant de foudre. A l'appui des moyens expérimentaux mis en œuvre pour les mesures du courant $I$ et de sa dérivée temporelle $\mathrm{d} I / \mathrm{d} t$ (Leteinturier, 1988), nous avons pu mesurer simultanément les signaux de courant et de champ électrique, soit à $50 \mathrm{~m}$, soit à $5 \mathrm{~km}$ de la décharge, selon les campagnes de mesures.

La figure 7 donne également l'enregistrement de $\mathrm{d} I / \mathrm{d} t$ obtenu simultanément à $\mathrm{d} E / \mathrm{d} t$ (à $50 \mathrm{~m}$ de la décharge) lors du $4^{c}$ arc-en-retour de l'éclair déclenché 8504 à Kennedy Space Center en 1985. Nous pouvons tout d'abord remarquer la similitude des formes d'onde, au moins pendant toute la phase transitoire du signal. Les valeurs crête obtenues sont $95 \mathrm{kA} / \mu \mathrm{s}$ et $110,5 \mathrm{kV} / \mathrm{m} / \mu \mathrm{s}$, la valeur crête du courant associée était $11,2 \mathrm{kA}$.

Pour chaque campagne de mesures (en 1985 et 1986, d $E / \mathrm{d} t$ était mesuré à $50 \mathrm{~m}$ et en 1987 à $5 \mathrm{~km}$ ), nous avons relié les valeurs crête des signaux $\mathrm{d} I / \mathrm{d} t$ et $\mathrm{d} E / \mathrm{d} t$. Nous remarquons sur la figure 9 que ces valeurs sont bien reliées linéairement pour chacune des années. Les droites de régression linéaire ont été tracées. Nous remarquons que les droites obtenues à partir des valeurs $\mathrm{d} E / \mathrm{d} t$ mesurées à $50 \mathrm{~m}$ de la décharge ont des pentes beaucoup plus élevées que lorsque $\mathrm{d} E / \mathrm{d} t$ était mesuré à $5 \mathrm{~km}$. La première explication provient de la contribution des composantes statique et induite du champ électrique à proche distance alors qu'à $5 \mathrm{~km}$ et pour les premiers instants de la décharge (lorsque $\mathrm{d} E / \mathrm{d} t$, ou $\mathrm{d} I / \mathrm{d} t$ atteignent leur valeur crête, l'impulsion de courant n'a encore parcouru que quelques dizaines de mètres au-dessus du sol), la composante rayonnée est la seule qui intervienne. La conductivité finie du sol $(\sigma<5 \mathrm{~S} / \mathrm{m})$ a pu entraîner l'atténuation des valeurs les plus élevées de $\mathrm{d} E / \mathrm{d} t$ à $5 \mathrm{~km}$ et ainsi diminuer la pente de la droite de régression linéaire et de plus empêcher qu'elle passe par l'origine.

Ces relations entre les paramètres $\mathrm{d} E / \mathrm{d} t$ et $\mathrm{d} I / \mathrm{d} t$ nous ont amené à les comparer avec celle 


\begin{tabular}{|c|c|c|c|c|c|c|}
\hline Type d'arc & \multicolumn{2}{|c|}{$\begin{array}{l}\mathrm{d} E / \mathrm{d} t_{\text {crête }} \\
(\mathrm{V} / \mathrm{m} / \mu \mathrm{s})\end{array}$} & \multicolumn{2}{|c|}{$\begin{array}{c}\text { DSMH } \\
\text { (ns) }\end{array}$} & \multicolumn{2}{|c|}{$\begin{array}{c}E_{\text {crête }} \\
(\mathrm{V} / \mathrm{m})\end{array}$} \\
\hline $\begin{array}{l}1^{\text {ers }} \\
\text { arcs-en-retour }\end{array}$ & $\begin{array}{c}N=62 \\
45,4 \\
\sigma=13,4\end{array}$ & $\begin{array}{c}N=49 \\
39,7 \\
\sigma=11,2\end{array}$ & $\begin{array}{c}N=64 \\
97,2 \\
\sigma=18,4\end{array}$ & $\begin{array}{c}N=49 \\
81 \\
\sigma=26\end{array}$ & $\begin{array}{c}N=48 \\
11,1 \\
\sigma=3,7\end{array}$ & $\begin{array}{c}N=48 \\
10,7 \\
\sigma=6,1\end{array}$ \\
\hline $\begin{array}{l}\text { arcs-en-retour } \\
\text { subséquents } \\
\text { classiques }\end{array}$ & $\begin{array}{c}N=6 \\
40,6 \\
\sigma=18,0\end{array}$ & $\begin{array}{c}N=10 \\
50,3 \\
\sigma=17,7\end{array}$ & $\begin{array}{c}N=7 \\
81,4 \\
\sigma=10,7\end{array}$ & $\begin{array}{l}N=10 \\
77 \\
\sigma=18\end{array}$ & $\begin{array}{c}N=5 \\
4,5 \\
\sigma=1,9\end{array}$ & $\begin{array}{c}N=10 \\
6,7 \\
\sigma=3,7\end{array}$ \\
\hline $\begin{array}{l}\text { arcs-en-retour } \\
\text { subséquents avec } \\
\text { précurseur par pas }\end{array}$ & $\begin{array}{c}N=7 \\
51,7 \\
\sigma=11,5\end{array}$ & $\begin{array}{c}N=17 \\
41 \\
\sigma=13,7\end{array}$ & $\begin{array}{c}N=7 \\
98,6 \\
\sigma=16,8\end{array}$ & $\begin{array}{l}N=17 \\
79 \\
\sigma=18\end{array}$ & $\begin{array}{c}N=6 \\
8,7 \\
\sigma=2,2\end{array}$ & $\begin{array}{c}N=17 \\
5,8 \\
\sigma=2,7\end{array}$ \\
\hline
\end{tabular}

\begin{tabular}{|c|c|c|c|c|c|}
\hline & \multicolumn{2}{|c|}{$\begin{array}{l}\mathrm{d} E / \mathrm{d} t_{\text {crête }} \\
(\mathrm{V} / \mathrm{m} / \mu \mathrm{s})\end{array}$} & \multicolumn{2}{|c|}{$\begin{array}{c}\text { DSMH } \\
\text { (ns) }\end{array}$} & $\begin{array}{c}E_{\text {crête }} \\
(\mathrm{V} / \mathrm{m}) \\
*\end{array}$ \\
\hline précurseurs par pas & $\begin{array}{c}N=17 \\
15,2 \\
\sigma=6,0\end{array}$ & & $\begin{array}{c}N=8 \\
68,8 \\
\sigma=6,4\end{array}$ & & \\
\hline intranuages & $\begin{array}{c}N=22 \\
9,2 \\
\sigma=5,4\end{array}$ & $\begin{array}{c}N=19 \\
13,0 \\
\sigma=4,0\end{array}$ & & $\begin{array}{c}N=19 \\
61 \\
\sigma=13\end{array}$ & $\begin{array}{c}N=19 \\
1,7 \\
\sigma=1,4\end{array}$ \\
\hline
\end{tabular}

Fig. 4. - Valeurs crête des signaux $\mathrm{d} E / \mathrm{d} t$ et $E$ (pic initial) normalisées à $100 \mathrm{~km}$; durée à mi-hauteur des signaux $\mathrm{d} E / \mathrm{d} t$, pour les arcs-en-retour (premiers ou subséquents), intranuages et précurseurs par pas. $N$ : nombre d'enregistrements ; $\sigma$ : écart-type ; $\left(^{*}\right)$ éclairs localisés à $35 \mathrm{~km}$ du site de mesures en 1984 (Leteinturier et al., 1985); $\left(^{+}\right)$éclairs localisés entre 15 et $40 \mathrm{~km}$ du site de mesures en 1985 (Bailey et al., 1988).

$[\mathrm{d} E / \mathrm{d} t$ and $E$ (initial peak) peak values normalized to $100 \mathrm{~km}$; full width at half maximum of the $\mathrm{d} E / \mathrm{d} t$ signals, for the return-strokes (first and subsequent), intracloud discharges and stepped-leaders. $N$ : number of events ; $\sigma:$ standarddeviation; $\left(^{*}\right)$ flashes located $35 \mathrm{~km}$ from the measuring site in 1984 (Leteinturier et al., 1985); $\left(^{+}\right)$flashes located between 15 and $40 \mathrm{~km}$ from the measuring site in 1985 (Bailey et al., 1988).]

donnée par le modèle de ligne de transmission (Uman et al., 1975) qui est définie de la façon suivante lorsque les composantes induite et statique sont négligeables par rapport à la composante rayonnée :

$$
\frac{\mathrm{d} E}{\mathrm{~d} t}\left(t+\frac{D}{c}\right)=-\frac{\mu_{0} V}{2 \pi D} \times \frac{\mathrm{d} I}{\mathrm{~d} t}(t)
$$

$\mu_{0}:$ permittivité du vide

$D$ : distance d'observation

$V$ : vitesse de propagation de l'onde de courant dans le canal.

Nous avons représenté cette relation sur la figure 9 (droite notée TLM) en supposant $V=1,3 \times$ $10^{8} \mathrm{~m} / \mathrm{s}$, valeur provenant de mesures optiques (Idone et al., 1984 ; Hubert-Mouget, 1981). En supposant l'équation précédente applicable pour nos mesures, nous déduisons des valeurs de $V$ plus grandes. Si nous corrigeons l'amplitude de $\mathrm{d} E / \mathrm{d} t$ $(50 \mathrm{~m})$ pour ne prendre en compte que la composante rayonnée (Leteinturier, 1988), nous obtenons pour les mesures faites en 1985 et $1986, V=2,4 \times$ $10^{8} \mathrm{~m} / \mathrm{s}$ en moyenne $\left(\sigma=0,34 \times 10^{8} \mathrm{~m} / \mathrm{s}\right)$ et pour les mesures faites en 1987: $V=1,9 \times 10^{8} \mathrm{~m} / \mathrm{s}$ en moyenne $\left(\sigma=0,2 \times 10^{8} \mathrm{~m} / \mathrm{s}\right)$, valeurs toutes plus élevées que celles obtenues par mesures optiques : Willett et al. (1988, a) donnent $1,28 \times 10^{8} \mathrm{~m} / \mathrm{s}$ $\left(\sigma=0,11 \times 10^{8} \mathrm{~m} / \mathrm{s}, N=7\right)$ pour 1985 et Willett et al. $(1988, \mathrm{~b})$ donnent $1,52 \times 10^{8} \mathrm{~m} / \mathrm{s}(\sigma=0,17 \times$ $\left.10^{8} \mathrm{~m} / \mathrm{s}, N=18\right)$ pour 1987.

Rappelons que les mesures optiques ont été faites sur des portions du canal de foudre à quelques centaines de mètres au-dessus du sol. Les mesures $\mathrm{de} \mathrm{d} I / \mathrm{d} t$ et $\mathrm{d} E / \mathrm{d} t$ font référence aux premières dizaines de mètres du canal au-dessus du sol. L'ensemble de ces résultats suggère que la vitesse est 


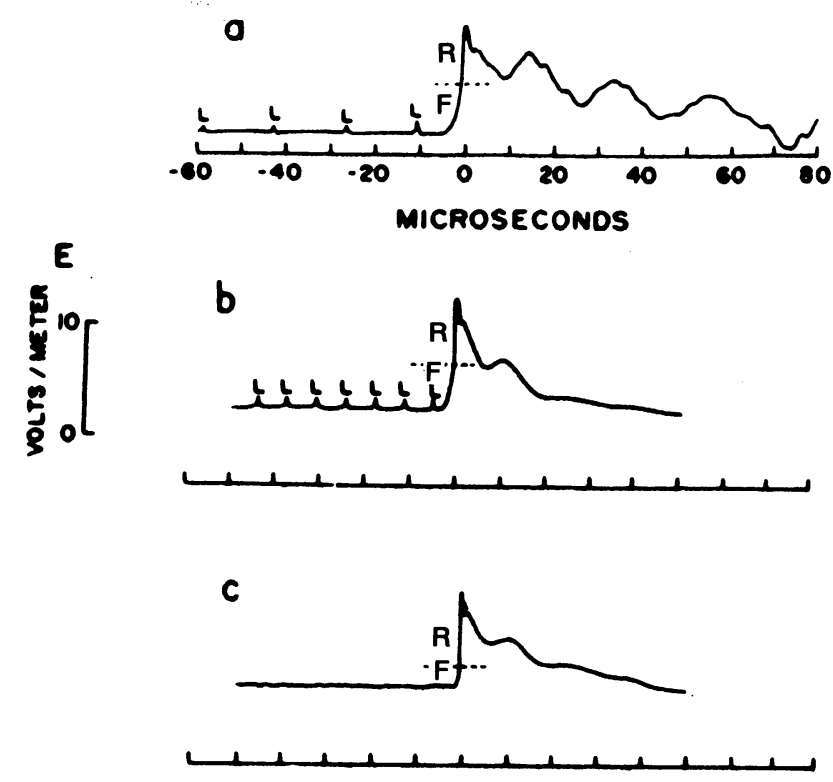

Fig. 5. - Formes d'onde typiques des signaux de champ électrique rayonnés lors des décharges nuage-sol, par un premier arc-en-retour (a), un arc-en-retour subséquent précédé d'un précurseur par pas (b), et un arc-en-retour subséquent précédé par un précurseur continu (c). Les amplitudes sont normalisées à $100 \mathrm{~km}$. Les petites impulsions « $\mathrm{L}$ », caractéristiques des pas des précurseurs sont suivies d'un front lent « $F$ » et d'une transition rapide « $\mathbf{R}$ ». Les courbes sont représentées avec une échelle des temps $10 \mu \mathrm{s} / \mathrm{div}$, d'après Weidman et Krider (1978).

[Typical waveforms of electric field signals radiated by a first return-stroke (a), a subsequent return-stroke, preceded by a stepped-leader (b), and a subsequent returnstroke preceded by a dart-leader (c). The magnitudes are normalized to $100 \mathrm{~km}$. The small pulses «L», due to stepped-leaders are followed by a «slow front $F$ » and a fast transition « $R$ ». The signals are plotted with a time scale $10 \mu \mathrm{s} /$ div., after Weidman-Krider (1978).]

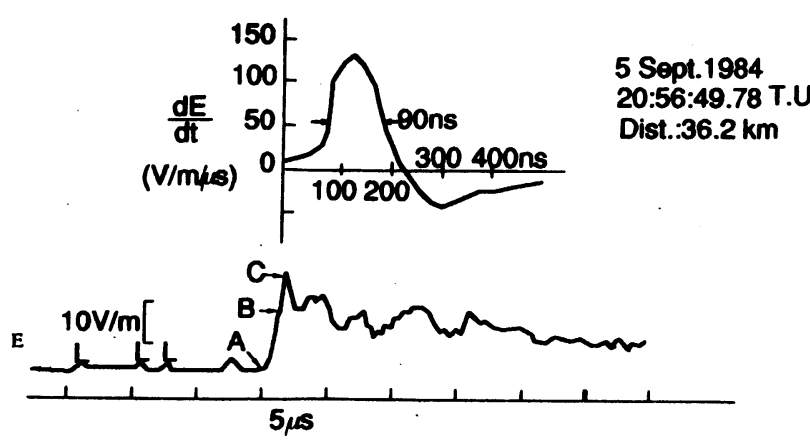

Fig. 6. - Exemple de signature $\mathrm{d} E / \mathrm{d} t$ (en haut), et $E$ (en bas) produites par un premier arc-en-retour à $36,2 \mathrm{~km} \mathrm{du}$ site de mesures.

[d $E / \mathrm{d} t$ waveform example (top), and $E$ waveform (bottom) produced by a first return-stroke, $36.2 \mathrm{~km}$ from the measuring site.]
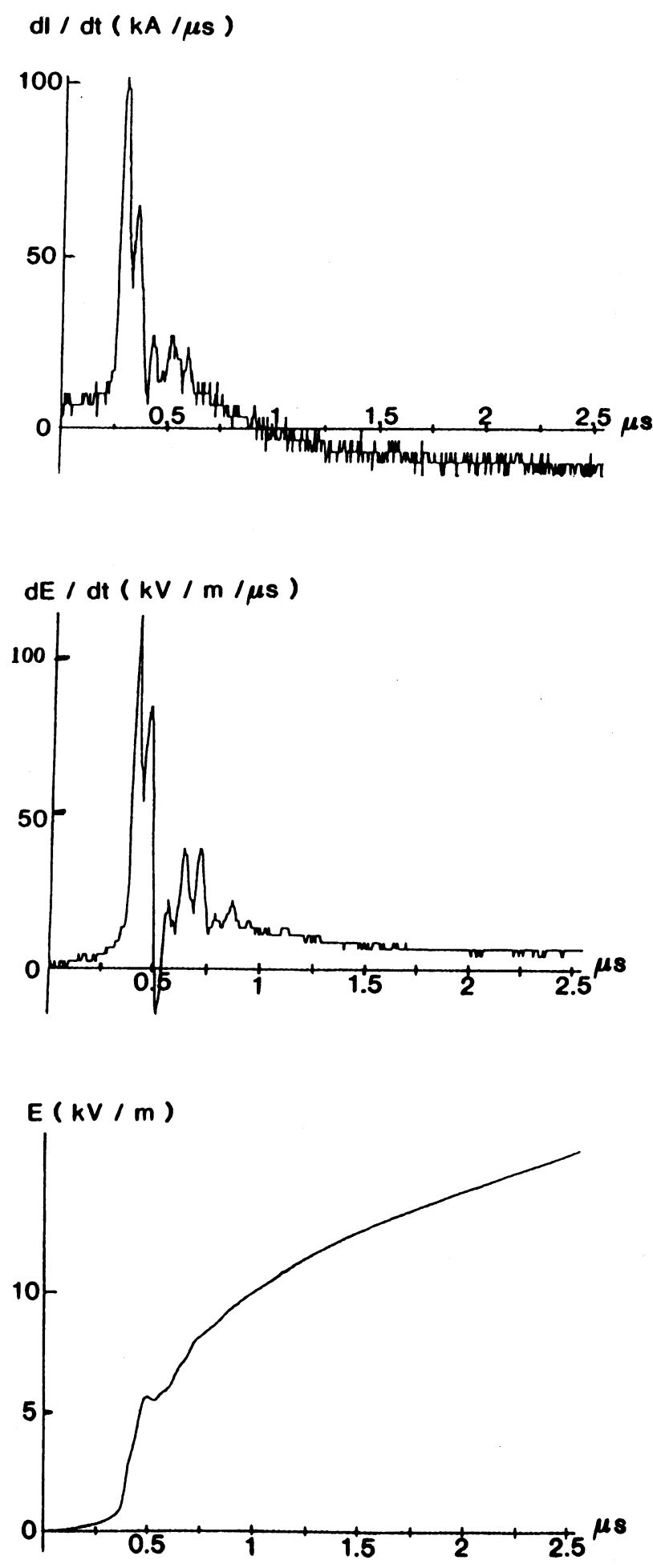

Fig. 7. - Signaux $\mathrm{d} I / \mathrm{d} t$ et $\mathrm{d} E / \mathrm{d} t$ enregistrés simultanément $(\mathrm{d} E / \mathrm{d} t$ à $50 \mathrm{~m})$ de l'éclair déclenché 8504 à Kennedy Space Center lors du $4^{\mathrm{e}}$ arc-en-retour. Champ électrique $E$ obtenu par intégration numérique. Le courant crête de l'arc-en-retour était $11,2 \mathrm{kA}$.

$[\mathrm{d} I / \mathrm{d} t$ and $\mathrm{d} E / \mathrm{d} t$ signals recorded simultaneously $(\mathrm{d} E / \mathrm{d} t, 50 \mathrm{~m}$ range) during the fourth stroke of the triggered flash, referenced 8504, at Kennedy Space Center. The $E$ field was obtained by a numerical integration. The peak current of that return-stroke was $11.2 \mathrm{kA}$.] 
beaucoup plus grande à la base du canal (supérieure à $2 \times 10^{8} \mathrm{~m} / \mathrm{s}$ ). Ce résultat est important pour l'amélioration des modèles, notamment pour remonter au courant de foudre à partir des mesures électromagnétiques.
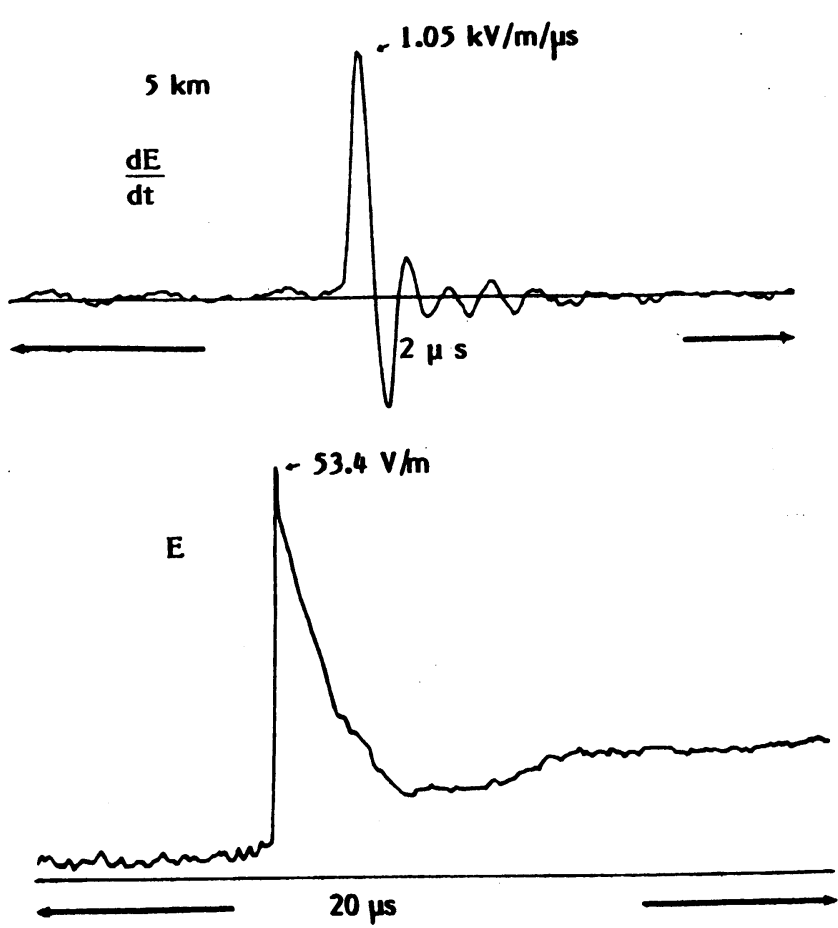

Fig. 8. - Enregistrement simultané des signaux $\mathrm{d} E / \mathrm{d} t$ et $E$ à $5 \mathrm{~km}$ de l'éclair déclenché 8717 lors du $3^{\mathrm{e}}$ arc-enretour. Le courant crête de l'arc-en-retour était $7 \mathrm{kA}$.

$[\mathrm{d} E / \mathrm{d} t$ and $E$ simultaneous pulses recorded during the third return-stroke of the triggered flash 8717 . The peak current was $7 \mathrm{kA}$.]
$D E / D T=F(D I / D T)$

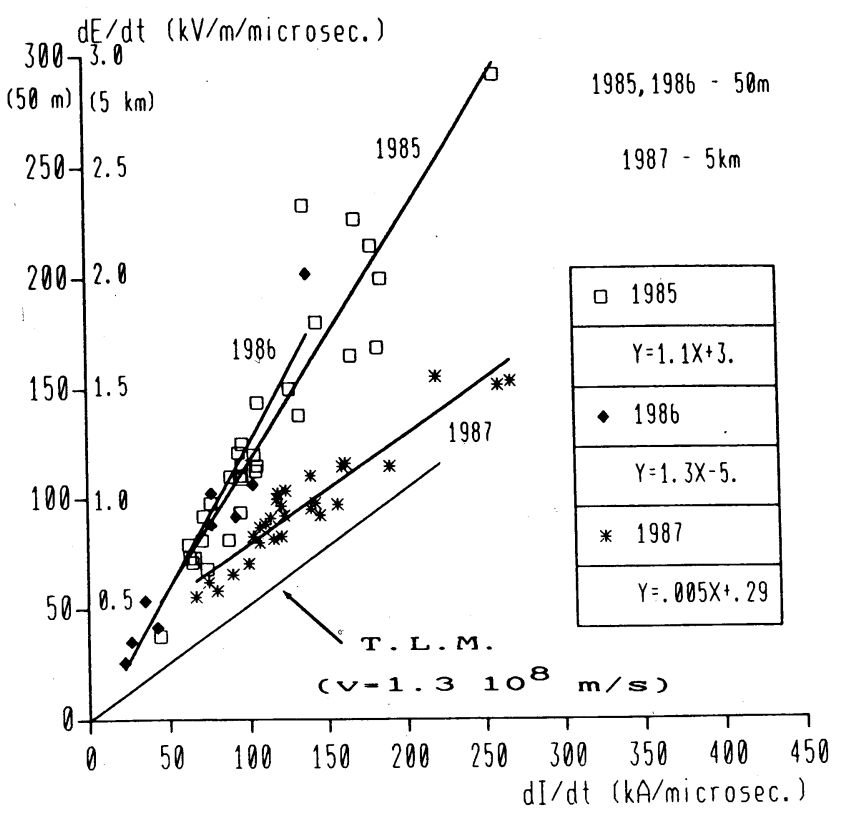

Fig. 9. - Relation entre les valeurs crête de la dérivée temporelle du courant de foudre mesurée à la base du canal et celles de la dérivée temporelle du champ électrique mesurée à $50 \mathrm{~m}$ de l'éclair en $1985(N=31)$ et 1986 $(N=9)$ et à $5 \mathrm{~km}$ en $1987(N=28)$. Tracé de la droite « TLM » reliant $\mathrm{d} E / \mathrm{d} t$ et $\mathrm{d} I / \mathrm{d} t$ par l'équation (1) avec $V=1,3 \times 10^{8} \mathrm{~m} / \mathrm{s}$.

[Relation between the peak values of $\mathrm{d} E / \mathrm{d} t$ and $\mathrm{d} I / \mathrm{d} t$, $\mathrm{d} I / \mathrm{d} t$ measured at the base of the lightning channel and $\mathrm{d} E / \mathrm{d} t 50 \mathrm{~m}$ from the flash in $1985(N=31)$ and in 1986 $(N=9)$ and $5 \mathrm{~km}$ from the flash in $1987(N=28)$. Linear regression fits are represented. The TLM line, which relates $\mathrm{d} I / \mathrm{d} t$ and $\mathrm{d} E / \mathrm{d} t$ by the equation (1) is also represented when $V=1.3 \times 10^{8} \mathrm{~m} / \mathrm{s}$.]

\section{Bibliographie}

Bailey J., Willett J. C., Krider E. P., Leteinturier C., Submicrosecond structure of the radiation fields from multiple events in lightning flashes, 8th Int. Conf. on Atmospheric Electricity, Uppsala, Suède (Juin 1988).

Hubert P., Mouget G., Return-stroke velocity measurements in two triggered lightning flashes, $J$. Geophys. Res. 86 (1981) 5253-5261.

Idone V., ORville R., Hubert P., Barret L., EYBERT-BERARD A., Correlated observations of three triggered lightning flashes, J. Geophys. Res. 89 (1984) 1385-1394.

Leteinturier C., Hamelin J., Analyse expérimentale des caractéristiques électromagnétiques des décharges orageuses dans la bande $200 \mathrm{~Hz}$ $20 \mathrm{MHz}$, Ann. Télécommun. tome $39 \mathrm{n}^{\circ}$ 5-6 (1984).
Leteinturier C., Weidman C., Hamelin J., Le BOULCH M., The phenomenology of natural and triggered thunderstorm discharges, 7th Int. Conf. on Atmospheric Electricity, Albany, N. Y. (1984).

Leteinturier C., Krider E. P., Willett J. C., Structure submicroseconde des champs rayonnés par la foudre, C.E.M., Clermont-Ferrand (1985).

LETEINTURIER C., Décharges orageuses : Analyse submicroseconde des courants et des champs électromagnétiques associés, Thèse de doctorat d'Etat, Université Paris VI (1988).

UMAN M. A., Lightning (Mc Graw-Hill Book Company) 1969.

UMAN M. A., Mc Lain D. K., KRider E. P., The electromagnetic radiation from a finite antenna, Am. J. Phys. Vol. 43 (Janvier 1975). 
Weidman C. D., Krider E. P., The fine structure of lightning return-stroke waveforms, J. Geophys. Res. 83 (1978) 6239-6247.

Weidman C. D., KRIDER E. P., The radiation field wave forms produced by intracloud lightning discharge processes, J. Geophys. Res. 84 (1979) 3159-3164.

Willett J. C., Idone V. P., ORVille R. E., Leteintu. RIER C., EYBERT-BERARD A., BARRET L., KRIDER E. P., An experimental test of the «transmission line model » of electromagnetic radiation from triggered lightning return-strokes, J. Geophys. Res. 93 (1988, a) 3867-3878.

Willett J. C., Bailey J. C., Idone V. P., ORville R. E., EYBERT-BERARD A., BARRET L., Submicrosecond intercomparison of radiation fields and currents in triggered lightning return-strokes based on the "transmission line model », 8th Conf. on Atmospheric Electricity, Proc., Uppsala, Suède (Juin 1988, b) pp. 539-544. 